\title{
Combined telovelar-minimal transvermian approach for a bleeding pontine cavernoma in a 48-year-old patient - case report and literature review
}

\author{
R. Radu ${ }^{1}$, G. Petrescu ${ }^{1}$, C. Gorgan ${ }^{2}$, C. Cristescu ${ }^{3}$, F. M. Brehar ${ }^{1,4}$, \\ R.M. Gorgan ${ }^{1,4}$ \\ ${ }^{1}$ Neurosurgery Department, "Bagdasar-Arseni” Emergency Hospital, Bucharest, ROMANIA \\ ${ }^{2}$ Neurology Department, "Bagdasar-Arseni” Emergency Hospital, Bucharest, ROMANIA \\ ${ }^{3}$ Anesthesiology and Intensive Care Department, "Bagdasar-Arseni" Emergency Hospital, \\ Bucharest, ROMANIA \\ ${ }^{4}$ Neurosurgery Department, “Carol Davila” University of Medicine and Pharmacy, ROMANIA
}

\begin{abstract}
Symptomatic pontine cavernous malformations carry a high risk of recurrent bleeding, which may result in permanent neurological deficit. Such lesions require surgical management that can be challenging to the neurosurgeon due to their anatomical location and their proximity to delicate neural structures. An ideal surgical approach should provide maximal surgical resection with minimal morbidity. We present the case of a 48-year-old woman with a pontine cavernoma with repeated spontaneous intralesional hemorrhages, resected using a telovelar approach extended by a minimal incision of the inferior vermis, with good surgical outcome. The telovelar approach provided a good access to the lateral recesses and the foramen of Luschka, while the lower vermian incision provided a greater vertical working angle inside the ventricle.
\end{abstract}

Key words: cavernous malformation, brainstem, suboccipital, telovelar, transvermian, motor evoked potentials

\section{Introduction}

A cavernous malformation is a cluster of thin-walled, angiogenically immature vessels that form blood-filled locules. Their prevalence in the general population is estimated at $0.1-0.5 \% \quad[1,2]$. Pontine cavernomas account for the majority of brainstem cavernous malformations [3]. It has been reported that infratentorial cavernomas are more dangerous than supratentorial lesions, with one report showing a 30 times greater tendency to bleed [4]. The characteristic lack of tight junctions 
between endothelial cells makes these lesions prone to leaking, most of them being surrounded by a complete hemosiderin rim. Cavernous malformations exhibit a wide variety of dynamic clinical behaviors, being a relatively common cause of recurrent spontaneous nontraumatic intracranial hemorrhage among young and middle-aged adults [5].

Once considered occult lesions due to their extremely low-flow angiographic behavior, without arteriovenous shunting, cavernous malformations are now easily distinguishable on CT and MRI. Based on their imaging appearance, Zabramski described four characteristic types of lesions [6]. Type I lesions appear hyperintense on both T1- and T2-weighted sequences due to subacute hemorrhage. Type II lesions contain loculated hemorrhages surrounded by a gliotic envelope, presenting as a mixed hyperintense/hypointense signal on both $\mathrm{T} 1$ and T2 imaging, with a low signal rim with blooming on $\mathrm{T}^{*}$ sequences. Type III lesions are iso- to hypointense, suggesting chronic hemorrhage. Type IV lesions are multiple punctate microhemorrhages visible only on $\mathrm{T}^{\star}$ sequences.

The management of asymptomatic lesions is often observation, while surgical treatment is indicated by most authors after the first symptomatic episode [7-9]. Due to the location of most brainstem cavernomas in the pontine and medullary regions, the posterior approaches are generally the most commonly used. These include the suboccipital approach, the supracerebellar infratentorial approach and the retrosigmoid approach. Other rare approaches include the telovelar approach, the transvermian approach and the far lateral transcondylar approach [10].

\section{Case presentation}

History

A 48-year-old woman with no prior medical history, presented to the emergency room with two weeks of progressive left-sided facial numbness, gait ataxia and intermittent diplopia, which was exacerbated by right lateral gaze.

The CT scan showed a hyperdense, homogenous mass with clearly demarcated contour in the right side of the pons (fig. 1).

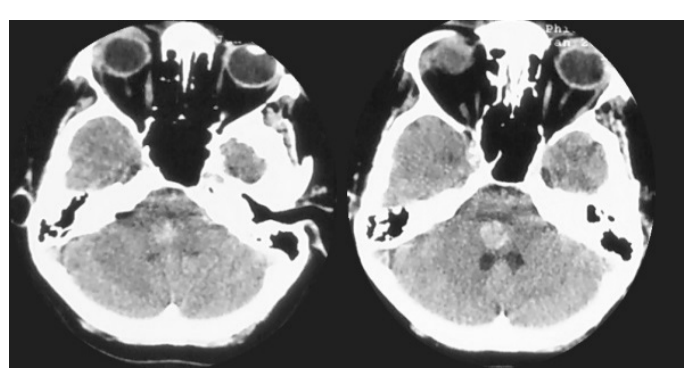

Figure 1 - Cerebral CT scan reveals a hyperdense mass with well-defined borders located in the right side of the pons.

MRI revealed a single non-contrast enhancing tumoral mass of $18 / 16 / 21 \mathrm{~mm}$, located in the right-middle pons, with inhomogenous structure due to $\mathrm{T} 1$ hyperintense areas suggestive of acute hemorrhage and a T1, T2 and FLAIR hypointense area (fig. 2). The mass was clearly contoured by a hypointense rim, suggestive of hemosiderin. MRI also showed minimal perilesional edema and mass effect on the fourth ventricle. The imaging was evocative of a Zabramski type II lesion. 


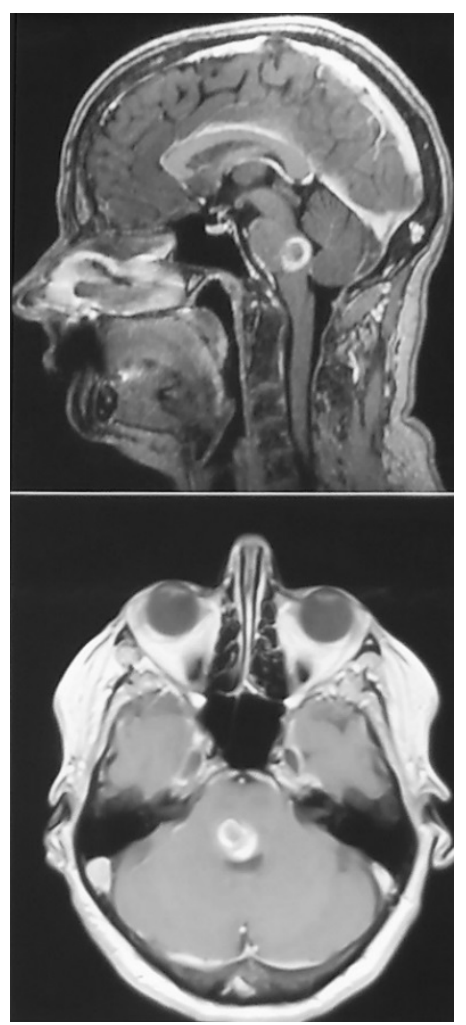

Figure 2 - MR image depicting a 18/16/21 mm mass suggestive of cavernoma.

\section{Surgical technique}

The patient was placed in a prone position with the head slightly raised and flexed for a better exposure of the space between the occiput and C1. A linear midline incision was performed at $1-2 \mathrm{~cm}$ above the external occipital protuberance down to the level of the C4 vertebra, followed by a median subocccipital craniectomy from below the torcula to the foramen magnum. The dura was opened with a Y-shaped flap over the cerebellar hemispheres. The approach was performed using microsurgical techniques. Due to the high risk of injury to the pontine nuclei, transcranial stimulation-induced motor and somatosensory evoked potentials were monitored intraoperatively.

The tela choroidea was incised at its attachment to the tinea of the fourth ventricle. The inferior medullary velum of the fourth ventricle was subsequently incised to complete the exposure to the foramen of Luschka.

An additional minimal incision of the inferior vermis expanded the surgical corridor, providing a better vertical working angle inside the fourth ventricle. An area of hemorrhagic effusion was visualized on the superior pontine aspect of the floor of the 4th ventricle. The cavity of the hematoma offered a good surgical corridor to the lesion.

Resection of the cavernoma proceeded by entering its capsule, followed by evacuation of its content and dissection in circumferential planes, leaving a cavity formed by normal parenchyma (fig. 3).

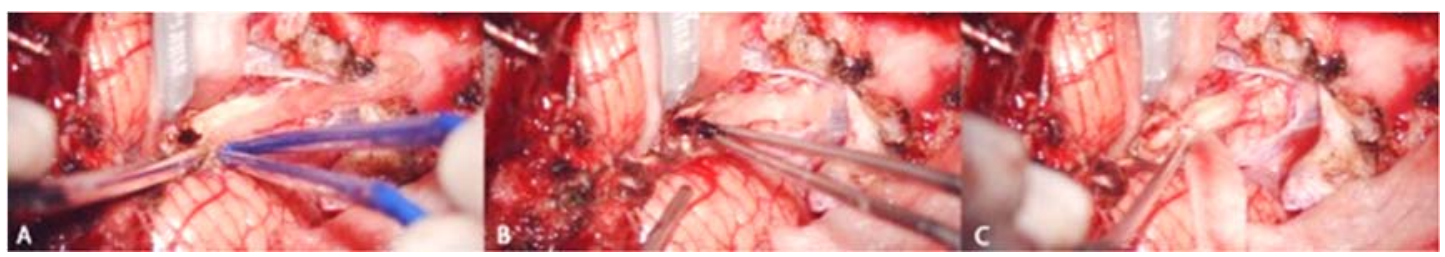

Figure 3 - Intraoperative view. A - Cavernoma surrounded by hemorrhagic effusion. B - Piecemeal resection of the cavernoma. C - Postresection cavity 


\section{Postoperative outcome}

Immediate postoperative exam showed no new-onset neurologic deficit and remission of the diplopia and facial numbness. Immediate postoperative CT showed gross total resection (fig. 4). The patient was discharged 11 days after surgery with no neurologic deficit (fig. 5).

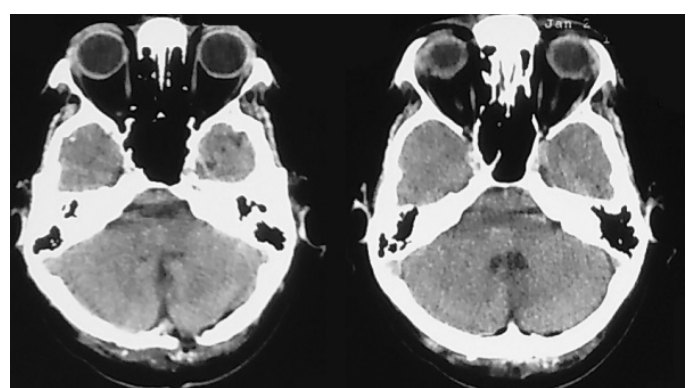

Figure 4 - Postoperative CT scan showing complete resection of the cavernoma.

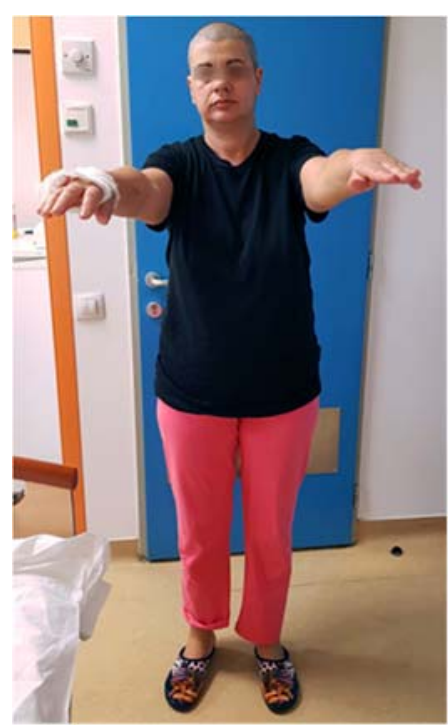

Figure 5 - The patient on postoperative day 7 with no neurologic deficit.

At the 2-month follow-up, the patient showed normal neurologic status (fig. 6) and complete resection was confirmed by MRI (fig. 7).

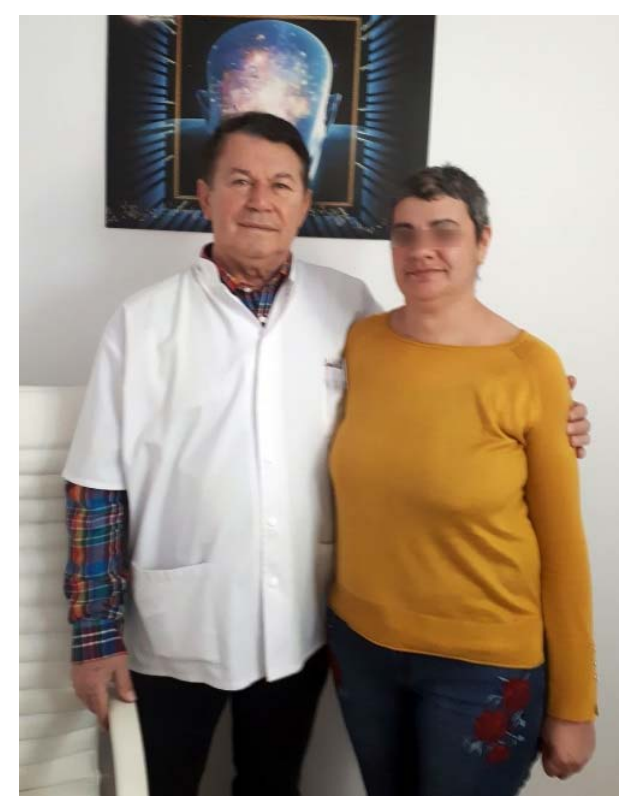

Figure 6 - Prof. R. M. Gorgan with the patient at the 2-month follow-up

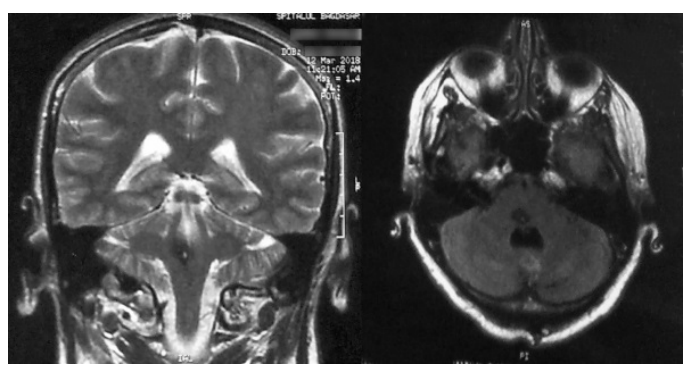

Figure 7 - MRI scan at the 2-month follow-up showing complete resection of the cavernoma

\section{Discussions}

Pontine cavernomas often represent a considerable microsurgical challenge to the operator, mainly because of the location, but also due to the high risk of complications. Surgery is indicated after severe or recurrent episodes of bleeding, neurologic worsening or when the cavernoma is exophytic and more accessible for the surgical resection $[11,12]$. 
Complete removal of the lesion is required in order to prevent recurring hemorrhagic events, as rebleeding has been shown to occur in $40 \%$ of cavernoma remnants after surgery [13].

Although cavernous malformations are now resected using less invasive approaches, it is still believed that exposure and adequate visualization are of utmost importance. Abla et al. recommend increased bone removal, within reason, to limit brain retraction and to enhance visibility [14].

The transvermian and telovelar approaches have been frequently reported in literature. The transvermian approach was used to resect the first cavernous malformation described in literature by Walter Dandy in 1928 [15]. However, recent reports describe transient mutism associated with incision of the vermis which resulted in abandoning this approach in favor of other routes to the fourth ventricle [16]. Another disadvantage of the transvermian approach is the risk of neural injury caused by the vermian incision, removal of the vermis or tonsils, and the retraction of two halves of the split vermis [17].

The telovelar and transvermian approaches to the fourth ventricle were compared by Tanriover et al [17]. These approaches were performed in 10 cadaveric specimens and the operative angle of approach and the superior, inferior, and lateral exposure for each approach were determined using Stealth image guidance with screen capture. The transvermian approach provided slightly better visualization of the medial aspect of the rostral fourth ventricle. The telovelar approach provided better access to the lateral recesses and foramen of

Luschka without an incision through cerebellum. The study did not determine area of exposure, which is an important consideration when working inside the fourth ventricle.

We decided to perfom a telovelar approach combined with an additional minimal linear incision of the inferior vermis. This approach offered an increased exposure of the superior pontine aspect of the fourth ventricle as well as greater working angle in the sagittal plane inside the ventricle compared to the telovelar approach alone while also avoiding the risks of a complete transvermian approach. The good surgical outcome with no intraoperative incidents and no neurological deficits suggests that the vermian incision may be safely performed if needed for a good surgical exposure.

\section{Conclusions}

The pons remains a difficult territory to access and mastery of the various surgical approaches is a difficult challenge for neurosurgeons. Most pontine cavernomas can be resected via the classically described approaches but it is possible to combine parts of various approaches in order to facilitate the resection of a lesion in its entirety while minimizing morbidity.

\section{Correspondence}

Brehar Felix Mircea

felixbrehar@yahoo.com 
210 | Radu et al - Combined telovelar-minimal transvermian approach for pontine cavernoma

\section{References}

1. Labauge P, Denier C, Bergametti F, Tournier-Lasserve E. Genetics of cavernous angiomas. Lancet Neurol. 2007;6(3):237-44.

2. Revencu N. Cerebral cavernous malformation: new molecular and clinical insights. J Med Genet. 2006;43(9):716-21.

3. Abla AA, Benet A, Lawton MT. The far lateral transpontomedullary sulcus approach to pontine cavernous malformations: Technical report and surgical results. Neurosurgery. 2014;10(3):472-80.

4. Porter PJ, Willinsky RA, Harper W, Wallace MC. Cerebral cavernous malformations: natural history and prognosis after clinical deterioration with or without hemorrhage. J Neurosurg. 1997;87(2):190-7.

5. Tatlisumak T, Cucchiara B, Kuroda S, Kasner SE, Putaala J. Nontraumatic intracerebral haemorrhage in young adults. Nat Rev Neurol. 2018.

6. Zabramski JM, Wascher TM, Spetzler RF, Johnson B, Golfinos J, Drayer BP, et al. The natural history of familial cavernous malformations: results of an ongoing study. J Neurosurg. 1994;80(3):422-32.

7. Wang CC, Liu A, Zhang JT, Sun B, Zhao YL. Surgical management of brain-stem cavernous malformations: Report of 137 cases. Surg Neurol. 2003;59(6):444-54.

8. Hauck EF, Barnett SL, White JA, Samson D. Symptomatic brainstem cavernomas. Neurosurgery. 2009;64(1):61-70.

9. Giovani A, Sandu A, Neacsu A, Gorgan RM. Surgical treatment and outcome of cerebral cavernomas - a 10 years' experience. Rom Neurosurg. 2014;21(4):395-405.
10. Hosainey SAM, Meling TR. A 34-year-old woman with brainstem cavernous malformation: The anterior transcallosal transchoroidal approach and literature review. J Neurol Surg Reports. 2014;75(2):e236-40.

11. Ohue S, Fukushima T, Kumon Y, Ohnishi T, Friedman AH. Surgical management of brainstem cavernomas: Selection of approaches and microsurgical techniques. Neurosurg Rev. 2010;33(3):315-22. 12. Abla AA, Lekovic GP, Turner JD, De Oliveira JG, Porter R, Spetzler RF. Advances in the treatment and outcome of brainstem cavernous malformation surgery: A single-center case series of 300 surgically treated patients. Neurosurgery. 2011;68(2):403-14.

13. Mouchtouris N, Chalouhi N, Chitale A, Starke RM, Tjoumakaris SI, Rosenwasser RH, et al. Management of cerebral cavernous malformations: from diagnosis to treatment. ScientificWorldJournal. 2015;2015:808314.

14. Abla AA, Turner JD, Mitha AP, Lekovic G, Spetzler RF. Surgical approaches to brainstem cavernous malformations. Neurosurg Focus. 2010;29(3):E8.

15. Dandy WE. Venous abnormalities and angiomas of the brain. Arch Surg. 1928 Nov 1;17(5):715

16. Deshmukh VR, Figueiredo EG, Deshmukh P, Crawford NR, Preul MC, Spetzler RF. Quantification and comparison of telovelar and transvermian approaches to the fourth ventricle. Neurosurgery. 2006;58(SUPPL. 2):202-7.

17. Tanriover N, Ulm AJ, Rhoton AL, Yasuda A. Comparison of the transvermian and telovelar approaches to the fourth ventricle. J Neurosurg. 2004;101:484-98. 